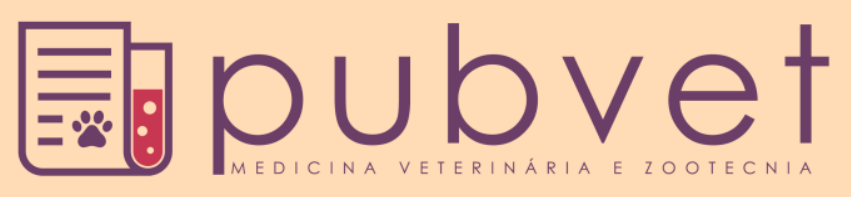

https://doi.org/10.22256/pubvet.v12n2a30.1-6

\title{
Ocorrência de Scomberocotyle scomberomori em Serra spanish mackerel do sudoeste do Oceano Atlântico
}

\author{
Cláudio Giovanio da Silva ${ }^{\bullet *}$, Naibe Cristina de Figueiredo ${ }^{2}$, José Ticiano Arruda \\ Ximenes de Lima 3 \\ ${ }^{1}$ Laboratório de Sanidade Aquática, Centro de Ciências Biológicas e da Saúde, Universidade Federal Rural do Semi-Árido, \\ Mossoró - RN, Brazil. E-mail: giovaniosl@bol.com.br \\ ${ }^{2}$ Pós-graduação em Produção Animal, Centro de Ciências Agrárias, Universidade Federal Rural do Semi-Árido, Mossoró - \\ RN, Brazil.E-mail: naibecristina@hotmail.com \\ ${ }^{3}$ Docente do Curso de Engenharia de Pesca, Centro de Ciências Agrárias, Universidade Federal Rural do Semi-Árido, \\ Mossoró - RN, Brazil. E-mail: ticiano.ximenes@ufersa.edu.br \\ *Autor para correspondência
}

RESUMO. O padrão de dispersão dos parasitas tem sido considerado de grande importância para a dinâmica populacional da relação hospedeiro-parasita. Este trabalho relata o primeiro registro do parasita monogenético Scomberocotyle scomberomori no hospedeiro Serra spanish mackerel, peixe da família Scombridae. Os resultados mostraram baixa prevalência $(4,7 \%)$ do parasita $S$. scomberomori na câmara branquial do novo hospedeiro (Serra spanish mackerel - Scomberomorus brasiliensis).

Palavras chave: Monogenea em Scombridae, parasito de peixe, peixe marinho

\section{Occurrence of Scomberocotyle scomberomori in Serra spanish mackerel from the southwest Atlantic Ocean}

ABSTRACT. The dispersion pattern of the parasites has been considered of great importance for the population dynamics of the host-parasite relationship. This work reports the first record of the monogenetic parasite Scomberocotyle scomberomori in the host Serra spanish mackerel, fish of the Scombridae family. The results showed a low prevalence $(4.70 \%)$ of the S. scomberomori parasite in the chamber branchial of the new host (Serra spanish mackerel - Scomberomorus brasiliensis).

Keywords: Monogenea in Scombridae, parasitized fish, marine fish

\section{Ocurrencia de Scomberocotyle scomberomori en Sierra española mackerel del suroeste del Océano Atlántico}

RESUMEN. El patrón de dispersión de los parásitos ha sido considerado de gran importancia para la dinámica poblacional de la relación hospedador-parásito. Este trabajo relata el primer registro del parásito monogenético Scomberocotyle scomberomori en el huésped Serra spanish mackerel, pescado de la familia Scombridae. Los resultados mostraron baja prevalencia $(4,70 \%)$ del parásito $S$. scomberomori en la cámara branquial del nuevo hospedador (Serra spanish mackerel - Scomberomorus brasiliensis).

Palabras clave: Monogenea en Scombridae, parasito de pescado, peces marinos 


\section{Introdução}

O padrão de dispersão dos parasitas tem sido considerado de grande importância para a dinâmica populacional da relação hospedeiroparasita (Penczykowski et al., 2016, Carvalho et al., 2017). São nos hospedeiros, que processos dependentes da abundância parasitária exercem influência sobre a sobrevivência ou fecundidade do hospedeiro (Visser et al., 2017).

Geralmente os danos causados ao hospedeiro por doenças parasitárias estão relacionados à espécie de parasito, sua forma de alimentação, órgão ou tecido do hospedeiro afetado, intensidade do parasitismo e ao estado geral do hospedeiro (Choi et al., 2014, Rynkiewicz et al., 2015). As infestações parasitárias podem retardar o crescimento e o ganho de peso dos peixes hospedeiros quando afetam a ingestão de alimentos (Barber et al., 2000, Bellay et al., 2012). De acordo com Carvalho et al. (2010) variações na composição de uma comunidade parasitária pode ser determinada por modificações nas condições ambientais e do hábitat dos hospedeiros que podem estar sujeitos à influência de possíveis ciclos sazonais.

As brânquias de Spanish Mackerels (Scomberomorus spp., fam. Scombridae) são conhecidas por estarem infestadas com representantes da família Thoracocotylidae (Rohde \& Hayward, 1999), esta família possui sete espécies em cinco gêneros, incluindo o gênero Scomberocotyle (Hayward \& Rohde, 1999a, Hayward \& Rohde, 1999b). Atualmente, o gênero Scomberocotyle possui apenas uma espécie descrita, Scomberocotyle scomberomori.

Este trabalho teve como objetivo relatar a primeira ocorrência de Scomberocotyle scomberomori em seu novo hospedeiro Serra Spanish Mackerel (Scomberomorus brasiliensis) do Sudoeste do Oceano Atlântico.

\section{Material e Métodos}

\section{Área de estudo e coleta dos peixes}

Os espécimes de Serra Spanish Mackerel, Scomberomorus brasiliensis Linnaeus, 1758 (Teleostei: Scombridae) foram amostrados durante o período de estudo na região pesqueira de Rio Grande do Norte, nordeste do Brasil. Todos os peixes foram submetidos à biometria, foram pesados em gramas $(\mathrm{g})$ e o comprimento foi medido em milímetros $(\mathrm{mm})$ e tiveram o sexo determinado de acordo com Vazzoler (1996).

\section{Coleta e identificação dos Parasitos}

Após captura na área em estudo e a realização da biometria, os peixes foram necropsiados para realização da investigação parasitaria. A coleta dos parasitos monogenéticos foi realizada seguindo as recomendações de Eiras et al. (2000) seguindo as orientações para identificação de monogenéticos. Os espécimes coletados foram conservados em álcool $70 \%$.

Após procedimentos iniciais, os monogenéticos fixados em álcool a $70 \%$ foram contados sob estereomicroscópio e identificados com auxílio de chaves de identificação propostas por Hendrix (1994) e Hayward \& Rohde (1999a).

Os parâmetros ecológicos de prevalência $(\mathrm{P} \%)$, intensidade media $(\mathrm{mI})$ e abundância media $(\mathrm{mA})$ de infestação parasitária foram calculados de acordo com Bush et al. (1997). Para determinar a preferência de micro-habitat da espécie de parasita, foram utilizados os dados de abundância e realizadas comparações entre os locais de infestação. Para estudo da ocorrência de parasitos e para verificar possível influência do sexo e dados biométricos (comprimento) dos hospedeiros na prevalência parasitária foi realizada a análise do teste $t$ de Student a 5\% de probabilidade $(\mathrm{P}<0,05)$ e a correlação de Pearson.

\section{Resultados}

Foram adquiridos 298 peixes Serra Spanish Mackerel ( $S$. brasiliensis) entre os meses de coleta. Os peixes apresentaram valores biométricos conforme a tabela 1. Durante a estação de verão (janeiro-março) foram adquiridos 112 exemplares de peixes, 66 no outono (abriljunho), 83 no inverno (julho-setembro) e 37 na primavera (outubro-dezembro). Houve uma predominância de machos nos meses em estudo (153 machos e 145 fêmeas). Todavia, não houve relação significativa entre o sexo e a infestação parasitaria $(\mathrm{P}>0,05)$.

Tabela 1. Valores mínimos (Min), máximos (Max), médios (Med) e desvio padrão (DP) da biometria (comprimento e peso total) da população de machos (đ̋) e fêmeas (ㅇ) de $S$. brasiliensis.

\begin{tabular}{lcccc}
\hline & \multicolumn{2}{c}{ Comprimento } & \multicolumn{2}{c}{ Peso total } \\
\hline \multirow{3}{*}{ Min } & $q$ & $\hat{1}$ & $q$ & $\widehat{\emptyset}$ \\
Max & 93,0 & 92,0 & 8,1 & 7,7 \\
\multirow{2}{*}{ Med \pm DP } & 805,0 & 661,0 & 3385,0 & 1493,5 \\
& $263,6 \pm$ & $281,9 \pm$ & $236,3 \pm$ & $219,3 \pm$ \\
& 157,2 & 143,8 & 465,8 & 279,9 \\
\hline
\end{tabular}


Entre os 298 peixes, 14 estavam parasitados no micro-habitat da câmara branquial. Este ectoparasito foi identificado como Scomberocotyle scomberomori (Figura 1) a partir de suas características morfológicas, com o corpo mais largo na margem posterior, gradualmente estreitando-se para a margem anterior. Haptor assimétrico, com extremidades livres (projetadas para fora do próprio corpo) e duas fileiras de grampos desiguais. Grampos geralmente mais largos do que longos. Presença de um par de hamuli. Esôfago com ramos laterais. Espinhos copulatório masculino de dois tipos, os posteriores menos numerosos e mais longos e retos. Vagina com dois pares de estruturas escleróticas semelhantes a um gancho. a

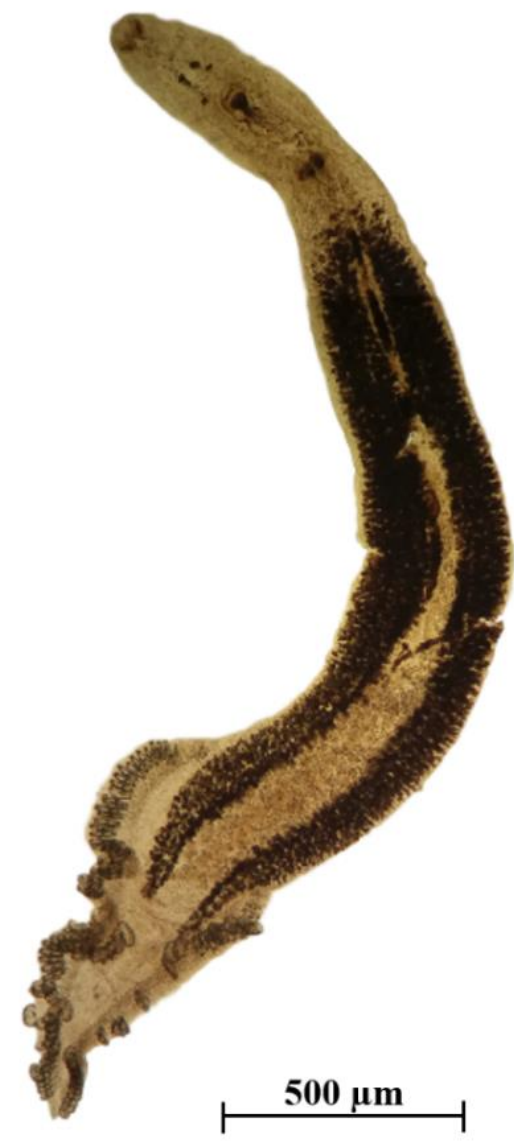

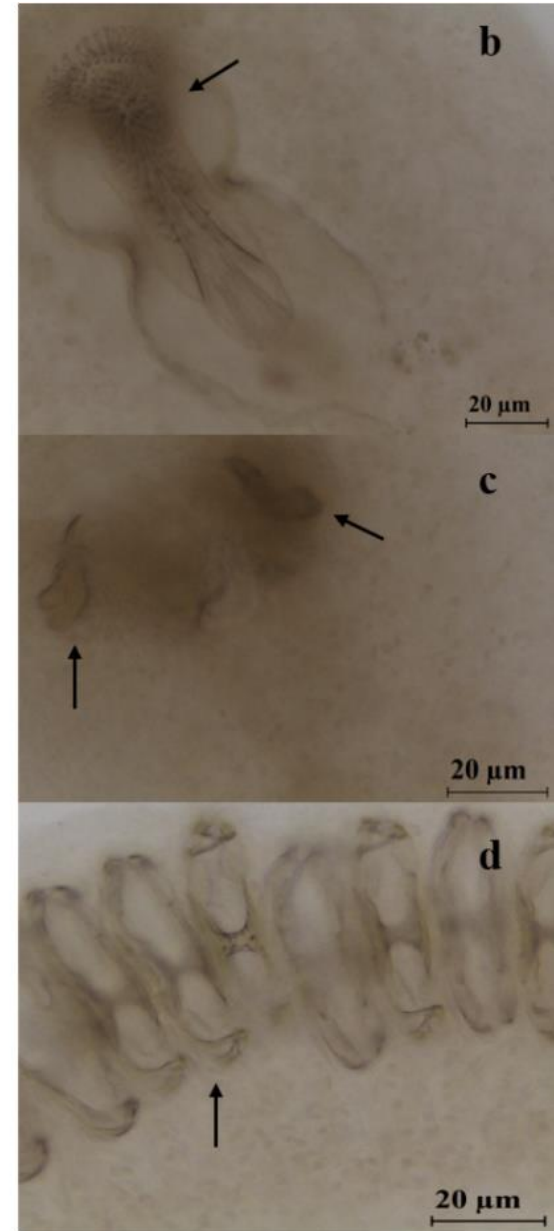

Figura 1. Ectoparasito monogenético Scomberocotyle scomberomori (a) Visão ventral, (b) Armadura copuladora masculina, (c) Armadura copuladora feminina e (d) Estrutura dos Grampos

Os índices ecológicos parasitários apresentaram uma prevalência geral $(\mathrm{P} \%=4,70)$, intensidade média geral $(\mathrm{mI}=1,79 \pm 0,97) \mathrm{e}$ abundância média geral $(\mathrm{mA}=0,10 \pm 0,49)$. A prevalência de Scomberocotyle scomberomori infestando Serra Spanish Mackerel por estações estão expressos na figura 2. Não houve uma variação significativa da prevalência entre as estações do ano $(\mathrm{P}>0,05)$; porém as estações de outono e primavera apresentaram uma maior prevalência, isto devido ao menor número de amostragem de hospedeiros, onde favoreceu o seu maior resultado.

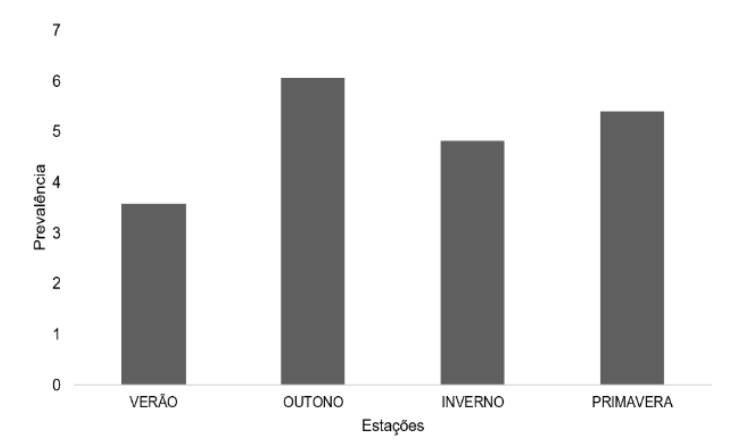

Figura 2. Prevalência de Scomberocotyle scomberomori em Serra Spanish Mackerel por estações do ano. 
A relação entre o parasito e os dados biométricos de comprimento está expressa na Figura 3. Não foram observadas diferenças $(\mathrm{P}>$ $0,05)$ em relação à ocorrência de $S$. scomberomori e a influência do comprimento de Serra spanish mackerel neste parasita, observada a partir do seu baixo valor de correlação $\left(R^{2}=0,097\right)$.

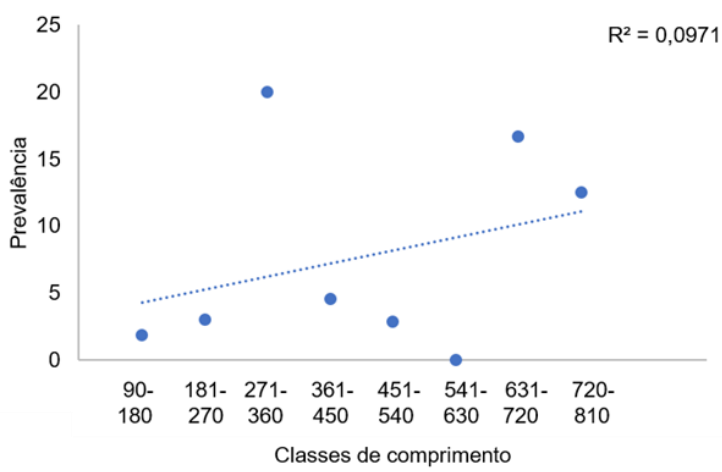

Figura 3. Regressão da relação entre a prevalência de Scomberocotyle scomberomori e o comprimento de Serra Spanish Mackerel.

\section{Discussão}

Este estudo relata pela primeira vez a ocorrência de Scomberocotyle scomberomori parasitando Serra Spanish Mackerel (Scomberomorus brasiliensis) no norte do Sudoeste do Oceano Atlântico. De acordo com Carvalho \& Luque (2009) estudos com hospedeiros naturalmente infestados são importantes para o conhecimento dos indicadores populacionais e registro de novos hospedeiros e novas áreas geográficas de espécies parasitárias.

A armadura do órgão copulatório masculino de S. scomberomori tem uma forte semelhança com a de Scomberomorocotyle munroi, mas esses dois se diferem claramente a partir de características importantes, como o número de vagina (o primeiro possui uma em comparação com o segundo que possui duas) e pares de hamuli (o primeiro possui um e o segundo possui dois).

Esta espécie de parasito já foi encontrada parasitando a maioria das espécies do gênero Scomberomorus, como S. maculatus na Florida, Carolina do Sul e México; S. cavala no Brasil e Colômbia; S. concolor no México; S. sierra na Colômbia, El Salvador, México e Panamá; $S$. tritor em Serra Leoa, Nigéria, Gana e Libéria (Hayward \& Rohde, 1999a). Hayward \& Rohde (1999a) já haviam realizado estudos parasitológicos em $S$. brasiliensis a procura do parasito Scomberocotyle scomberomori, mas não foram registrados indivíduos desta espécie.

Neste estudo, a relação parasitária de $S$. scomberomori com os dados biométricos de comprimento de Serra Spanish Mackerel não foi significativa $\left(R^{2}=0,097\right)$. Segundo Schalch et al. (2006) em hábitat em que há constância na temperatura da água, como nas regiões intertropicais, as variações sazonais do parasitismo não são significativas. No entanto, foi possível observar que as maiores prevalências parasitárias neste estudo ocorreram nas estações de outono e primavera.

Em relação às maiores prevalências nas estações de outono e primavera, isto ocorreu devido ao menor número de amostragem de hospedeiros nessas duas épocas $\left(\begin{array}{lll}66 & \text { e } & 37\end{array}\right.$ respectivamente), favorecendo o seu maior resultado devido este cálculo estar diretamente relacionado ao número de exemplares da amostra.

A abundancia média $(\mathrm{mA}=0,10)$ de $S$. scomberomori em Serra Spanish Mackerel está entre os valores de outras espécies de hospedeiros do gênero Scomberomorus que este parasito infestou, as abundancias destes variaram de 0,06 a 1,75 .

Bullard et al. (2003) mencionaram que o comportamento de formar cardumes facilita a dispersão horizontal das larvas de monogenéticos em algumas espécies de peixes. Neste sentido, os índices ecológicos de S. scomberomori apontam que este fato comportamental, no presente trabalha com Serra Spanish Mackerel $(S$. brasiliensis) influenciou a transmissão horizontal da espécie parasitária em estudo comprovada pela não relação significativa com os dados biométricos.

SegundoPavanelli et al. (2008) e Appeltans et al. (2012) diversos estudos têm sido realizados ao longo dos anos para determinar a diversidade relativa e o efeito do parasitismo no mundo. Porém, há poucas informações sobre os parasitos, principalmente monogenéticos em Serra Spanish Mackerel e em outras espécies da costa de Rio Grande do Norte.

Em conclusão, este estudo relata a primeira ocorrência do metazoário parasito monogenético Scomberocotyle scomberomori infestando um novo hospedeiro, Serra Spanish Mackerel (Scomberomorus brasiliensis) no norte do Sudoeste do Oceano Atlântico. 


\section{Agradecimentos.}

Os autores agradecem ao CNPQ (Conselho Nacional de Desenvolvimento Científico e Tecnológico) e CAPES (Coordenação de Aperfeiçoamento de Pessoal de Nível Superior) por auxiliar na pesquisa com apoio de bolsas de iniciação científica e pós-graduação.

\section{Referências Bibliográficas}

Appeltans, W., Ahyong, S. T., Anderson, G., Angel, M. V., Artois, T., Bailly, N., Bamber, R., Barber, A., Bartsch, I. \& Berta, A. 2012. The magnitude of global marine species diversity. Current Biology, 22, 2189-2202.

Barber, I., Hoare, D. \& Krause, J. 2000. Effects of parasites on fish behaviour: a review and evolutionary perspective. Reviews in Fish Biology and Fisheries, 10, 131-165.

Bellay, S., Ueda, B. H., Takemoto, R. M., Lizama, M. d. 1. A. P. \& Pavanelli, G. C. 2012. Fauna parasitária de Geophagus brasiliensis (Perciformes: Cichlidae) em reservatórios do estado do Paraná, Brasil. Revista Brasileira de Biociências, 10, 4-78.

Bullard, S. A., Goldstein, R. J., Hocking, R. \& Jewell, J. 2003. A new geographic locality and three new host records for Neobenedenia melleni (MacCallum)(Monogenea: Capsalidae). Gulf and Caribbean Research, $15,1-4$.

Bush, A. O., Lafferty, K. D., Lotz, J. M. \& Shostak, A. W. 1997. Parasitology meets ecology on its own terms: Margolis et al. revisited. The Journal of Parasitology, 83, 575-583.

Carvalho, A. R. \& Luque, J. L. 2009. Occurrence of Neobenedenia melleni (Monogenea: Capsalidae) in Trichiurus lepturus (Perciformes: Trichiuridae), naturally infested off coastal zone of Rio de Janeiro, Brazil. Revista Brasileira de Parasitologia Veterinária, 18, 74-76.

Carvalho, A. R., Martins, R. T., Bellei, P. M. \& Souza Lima, S. 2017. Aspectos ecológicos da helmintofauna de Hoplias malabaricus (Bloch, 1794)(Characiformes, Erythrinidae) da Represa Dr. João Penido (Juiz de Fora-MG, Brasil). Revista Brasileira de Zoociências, 18, 7-20.

Carvalho, A. R., Tavares, L. E. R. \& Luque, J. L. 2010. Variação sazonal dos metazoários parasitos de Geophagus brasiliensis
(Perciformes: Cichlidae) no rio Guandu, Estado do Rio de Janeiro, Brasil. Acta Scientiarum. Biological Sciences, 32, 159-167.

Choi, Y.-J., Aliota, M. T., Mayhew, G. F., Erickson, S. M. \& Christensen, B. M. 2014. Dual RNA-seq of parasite and host reveals gene expression dynamics during filarial worm-mosquito interactions. PLoS Neglected Tropical Diseases, 8, e2905.

Eiras, J. C., Takemoto, R. M. \& Pavanelli, G. C. 2000. Métodos de estudo e técnicas laboratoriais em parasitologia de peixes. Eduem, Maringá, Paraná.

Hayward, C. J. \& Rohde, K. 1999a. Revision of the monogenean subfamily Neothoracocotylinae Lebedev, 1969 (Polyopisthocotylea: Thoracocotylidae). Systematic Parasitology, 44, 183-191.

Hayward, C. J. \& Rohde, K. 1999b. Revision of the monogenean subfamily Thoracocotylinae Price, $\quad 1936 \quad$ (Polyopisthocotylea: Thoracocotylidae), with the description of a new species of the genus Pseudothoracocotyla Yamaguti, 1963. Systematic Parasitology, 44, 157-169.

Hendrix, S. S. 1994. Marine Flora and Fauna of the Eastern United States: Platyhelminthes: Monogenea. NOAA Tech. Rep., 121, 108.

Pavanelli, G. C., Eiras, J. C. \& Takemoto, R. M. 2008. Doenças de peixes: profilaxia, diagnóstico e tratamento. Eduem, Maringá.

Penczykowski, R. M., Laine, A. L. \& Koskella, B. 2016. Understanding the ecology and evolution of host-parasite interactions across scales. Evolutionary Applications, 9, 37-52.

Rohde, K. \& Hayward, C. J. 1999. Revision of the monogenean subfamily Priceinae Chauhan, 1953 (Polyopisthocotylea: Thoracocotylidae). Systematic Parasitology, 44, 171-182.

Rynkiewicz, E. C., Pedersen, A. B. \& Fenton, A. 2015. An ecosystem approach to understanding and managing within-host parasite community dynamics. Trends in Parasitology, 31, 212-221.

Schalch, S. H. C., Moraes, F. R. \& Moraes, J. R. E. 2006. Efeitos do parasitismo sobre a estrutura branquial de Leporinus macrocephalus Garavello e Britsk, 1988 (Anastomidae) e Piaractus mesopotamicus Holmberg, 1887 (Osteichthyes: Characidae). Revista Brasileira de Parasitologia Veterinária, 15, 110-115. 
Vazzoler, A. E. A. M. 1996. Biologia da reprodução de peixes teleósteos: teoria e prática. Eduem, Maringá, Paraná.

Visser, M. D., Schnitzer, S. A., Muller-Landau, H. C., Jongejans, E., Kroon, H., Comita, L. S., Hubbell, S. P. \& Wright, S. J. 2017. Tree species vary widely in their tolerance for liana infestation: a case study of differential host response to generalist parasites. Journal of Ecology.
Article History:

Received 10 October 2017

Accepted 28 October 2017

Available online 11 January 2018

License information: This is an open-access article distributed under the terms of the Creative Commons Attribution License 4.0, which permits unrestricted use, distribution, and reproduction in any medium, provided the original work is properly cited. 